\title{
Extremely low-volume, high-intensity interval training improves exercise capacity and increases mitochondrial protein content in human skeletal muscle
}

\author{
Jasmin K. Ma ${ }^{1^{*}}$, Trisha D. Scribbans ${ }^{1 *}$, Brittany A. Edgett ${ }^{1}$, J. Colin Boyd $^{1}$, Craig A. Simpson ${ }^{2}$, \\ Jonathan P. Little ${ }^{3}$, Brendon J. Gurd ${ }^{1}$ \\ ${ }^{1}$ Department of Kinesiology, Queen's University, Kingston, Canada \\ ${ }^{2}$ Department of Emergency Medicine, Kingston General Hospital, Kingston, Canada \\ ${ }^{3}$ Department of Health and Social Development, University of British Columbia Okanagan, Kelowna, Canada \\ Email: gurdb@queensu.ca
}

Received 7 July 2013; revised 7 August 2013; accepted 14 August 2013

Copyright (C) 2013 Jasmin K. Ma et al. This is an open access article distributed under the Creative Commons Attribution License, which permits unrestricted use, distribution, and reproduction in any medium, provided the original work is properly cited.

\begin{abstract}
Purpose: The purpose of the present study was to evaluate whole-muscle content of several proteins involved in the regulation of skeletal muscle mitochondrial protein content and anaerobic capacity following 4 weeks of extremely low-volume high-intensity interval training (HIT). Methods: Young, healthy, recreationally active adult males $(n=8)$ trained 4 times a week for 4 weeks on a cycle ergometer. Each session involved $4 \mathrm{~min}$ of total exercise comprised of eight $20 \mathrm{~s}$ intervals at $\sim \mathbf{1 7 0 \%}$ of peak aerobic power separated by $10 \mathrm{~s}$ rest. Muscle biopsies were taken prior to (pre) and $\sim 72$ hrs post-training (post). Participants completed an incremental peak oxygen uptake $\left(\mathrm{VO}_{2}\right.$ peak) test and a Wingate test pre-, mid-, and post-training. Results: $\mathrm{VO}_{2}$ peak was elevated $(p<$ $0.05)$ at mid- $(p<0.05)$ and post-training (pre: $40.5 \pm$ $3.8 \mathrm{ml} \cdot \mathrm{kg}^{-1} \cdot \mathrm{min}^{-1}$, mid: $43.4 \pm 2.5 \mathrm{ml} \cdot \mathrm{kg}^{-1} \cdot \mathrm{min}^{-1}$, post-: $47.2 \pm 2.9 \mathrm{ml} \cdot \mathrm{kg}^{-1} \cdot \mathrm{min}^{-1}$ ). Wingate mean power also increased with training (pre-: $701.0 \pm 73.0 \mathrm{~W}$, mid-: $\mathbf{7 4 5 . 5} \pm \mathbf{7 3 . 3} \mathrm{W}$, post-: $786.8 \pm \mathbf{8 0 . 0} \mathrm{W}$ ). While maximal citrate synthase activity was unchanged, protein expression of the mitochondrial protein cytochrome c oxidase (COX) subunit I $(+27 \% ; p<0.05)$ and COX IV $(+26 \% ; p<0.05)$, PDK2 $(+28 \% ; p=0.08)$ increased. Increases $(p<0.05)$ in both total PGC-1 $\alpha$ $(+19 \%)$, and nuclear PGC-1 $\alpha(+46 \%)$ were also observed after 4 weeks of HIT. No changes were observed in the whole-muscle contents of PDHe1a, PDK4, SIRT1, mTOR, S6K1, MCT1, or PFK protein. Conclusions: These results demonstrate that several mitochondrial protein (but not citrate synthase activ-
\end{abstract}

\footnotetext{
*These authors contributed equally to manuscript.
}

ity), PGC-1 $\alpha$ protein content, and exercise capacity can be improved in only $4 \mathrm{~min}$ of total training time per day, 4 days per wk using HIT cycle training.

Keywords: PGC-1 $\alpha$; Mitochondrial Biogenesis;

High-Intensity Interval Training; SIRT1; Aerobic Fitness

\section{INTRODUCTION}

Improved exercise tolerance and increases in mitochondrial protein in skeletal muscle have been demonstrated following high-intensity interval training (HIT) [1,2]. At present, the available evidence suggests that increases in exercise tolerance and mitochondrial protein following short-term endurance training and HIT are similar $[1,3,4]$. Assuming equivalent adaptation, HIT represents an alternative to endurance training requiring significantly less exercise time [1-3]. However, while minimal doses of maximal intensity HIT (2 - 3 min of exercise time per training session) induce significant adaptation $[1,2,5]$, these protocols require long periods of recovery between intervals such that total training time is still considerable ( 20 - $30 \mathrm{~min})$. Whether less intense interval protocols requiring less total training time produce similar increases in skeletal muscle mitochondrial content is not known.

The HIT protocol presented by Tabata et al. (1996), subsequently termed the "Tabata protocol", consists of eight 20 s intervals at $\sim 170 \%$ of maximal aerobic power separated by $10 \mathrm{~s}$ of rest. This extremely low-volume protocol totals less than $3 \mathrm{~min}$ of exercise time and only 4 min total training commitment per training session. In humans, training with this protocol improves both aerobic and anaerobic exercise capacity, and muscle endur- 
ance [6,7]. However, the mechanism(s) by which this minimal dose of exercise induces improvements in both aerobic and anaerobic capacity in humans is currently unclear.

While adapting the Tabata protocol to swimming in rats does improve markers of oxidative capacity $[4,8]$ whether the Tabata protocol improves mitochondrial protein content in human skeletal muscle, an adaptation that could contribute to increased aerobic capacity, is not known. The impact of the Tabata protocol on postulated regulators of skeletal muscle transcriptional (peroxisome proliferator-activated receptor $\gamma$ coactivator-1 alpha [PGC$1 \alpha$ ], sirtuin 1 [SIRT1]), and translational (mammalian target of rapamycin [mTOR] and ribosomal protein S6 kinase [S6K1]) control of mitochondrial biogenesis has also not been examined in human skeletal muscle. In addition to the mechanisms underlying aerobic adaptations following HIT training, the mechanisms that underlie the observed increase in anaerobic capacity induced by the Tabata protocol are also of interest. Specifically whether adaptations typically associated with improved anaerobic performance, including increased glycolytic capacity [9] and lactate transport [10], are also improved following extremely low-volume HIT is unknown.

The purpose of this study was twofold: 1) to confirm the rapid improvements in aerobic and anaerobic capacity previously observed following extremely low-volume HIT utilizing the Tabata protocol; and 2) to examine changes in markers of skeletal muscle mitochondrial protein, proteins involved in the regulation of mitochondrial biogenesis, and protein associated with skeletal muscle glycolytic capacity. We hypothesized that exercise capacity $\left(\mathrm{VO}_{2}\right.$ peak and Wingate anaerobic test), markers of mitochondrial content, and protein associated with glycolytic capacity would all increase following 4 weeks of Tabata-based cycle training.

\section{METHODS}

The training intervention for this study involved 4 wks of HIT using the "Tabata protocol" [7] 4 times per wk. Muscle biopsies were obtained pre- and post-training, while assessments of aerobic and anaerobic capacities were performed at pre-, mid- and post-training. Pretesting was completed the wk prior to the beginning of training and post-testing was completed $72 \mathrm{hr}$ following the final training session. Although we did not explicitly study a control group, we have previously shown that $\mathrm{VO}_{2}$ peak and several markers of muscular performance are unchanged over 4 wks when recreationally active university students are assigned to a control group [6].

Ten recreationally active males $(20.6 \pm 1.6 \mathrm{y} ; 182 \pm 7$ $\mathrm{cm} ; 79.7 \pm 15.6 \mathrm{~kg} ; \mathrm{VO}_{2}$ peak, $40.5 \pm 3.8 \mathrm{~mL} / \mathrm{min} / \mathrm{kg}$; peak power $975 \pm 114 \mathrm{~W}$ ) studying at Queen's University volunteered to participate in the study. Two participants did not complete the study due to conflicts outside of the study; all data associated with these participants were excluded. The remaining participants $(n=8)$ completed all 16 training sessions over the 4 wk period. The study was approved by the Health Sciences Human Research Ethics Board at Queen's University and all participants provided written informed consent.

\subsection{Training Protocol}

The training protocol utilized has been described previously [7]. Briefly, participants completed eight $20 \mathrm{~s}$ intervals separated by $10 \mathrm{~s}$ of rest on each training day. Intervals were completed on a Monark Ergomedic $874 \mathrm{E}$ stationary bicycle ergometer (Vansbro, Sweden) with a weight corresponding to $170 \%$ of peak aerobic power at $100 \mathrm{rpm}$. During rest periods participants cycled with no load at a cadence of their choice. Participants trained 4 times per wk for $4 \mathrm{wks}$, amounting to a total exercise time of $43 \mathrm{~min}$ (160 s per training session) and a total training time commitment, including $10 \mathrm{~s}$ rest periods, of $64 \mathrm{~min}$ (i.e. $16 \mathrm{~min}$ of total exercise time commitment per wk). During each participant's first training session heart rate (Polar Team2 Pro, Kempele, Finland) and work rate were monitored continuously while acute affect (Feeling Scale; a single item validated 11-point Likert bipolar FS ranged from -5 [Very Bad] to +5 [Very Good]), telic state (Felt Arousal Scale; scales from 1 to 6 with anchors provided at 1 [low arousal] and 6 [high arousal]) and perceived exertion (Borg) were assessed at rest, after the 4th and 8th interval, and $10 \mathrm{~min}$ into recovery.

\subsection{Physiological Testing}

Participants arrived at the first laboratory visit in a fasted state. One hr following a standardized breakfast, muscle biopsies were performed under sterile conditions with local anaesthesia (2\% lidocaine) using a Bergstrom biopsy needle [11] adapted with suction. Muscle tissue was immediately frozen in liquid nitrogen, and stored at $-80^{\circ} \mathrm{C}$ until analysis.

Forty-eight hr following the muscle biopsy (pre- and post-testing) and $72 \mathrm{hr}$ after the final training session of wk 2 (mid-testing), participants reported to the lab for a $\mathrm{VO}_{2}$ peak and Wingate cycling test. The $\mathrm{VO}_{2}$ peak ramp protocol consisted of 5 min of load-less cycling (Monark, Ergomedic 874 E, Vansbro, Sweden) followed by step increases in work rate of $25 \mathrm{~W} \cdot \mathrm{min}^{-1}$ to volitional fatigue. Gas exchange was measured throughout the protocol with a metabolic cart (Moxus AEI Technologies, Pittsburgh, PA). $\mathrm{VO}_{2}$ peak was calculated by averaging $\mathrm{VO}_{2}$ values from the final $30 \mathrm{~s}$ of the test. Following comple- 
tion of the $\mathrm{VO}_{2}$ peak test, participants rested for $30 \mathrm{~min}$ before completing a Wingate test.

\subsection{Muscle Analysis}

To prepare whole muscle lysates, frozen muscle tissue $(\sim 30-40 \mathrm{mg})$ was homogenized in $1 \mathrm{~mL}$ of ice-cold buffer containing: $50 \mathrm{mM}$ Tris- $\mathrm{HCl}$ (pH 7.4), 1\% Triton $\mathrm{X}-100,1 \mathrm{mM}$ of EDTA and EGTA, $50 \mathrm{mM} \mathrm{NaF}, 10 \mathrm{mM}$ sodium b-glycerophosphate, $5 \mathrm{mM}$ sodium pyrophosphate, $2 \mathrm{mM}$ DTT and Halt Protease Inhibitor Cocktail (100X, Thermo Fisher Scientific, Rockford, IL). Nuclei were isolated from $30-40 \mathrm{mg}$ of muscle tissue using a commercially available nuclear extraction kit (Pierce Biotechnology, Rockford, IL) as previously described [12]. Purity of nuclear extracts was confirmed via Western blot for cytosolic lactate dehydrogenase (LDH) and nuclear histone 3 (H3).

Protein contents were determined for whole muscle and nuclear extracts and equal amounts of total protein were loaded and separated by SDS-PAGE using $8 \%$, (SIRT1, PGC- $1 \alpha$, mTOR, S6K1, GAPDH), or $10 \%(\mathrm{H} 3$, LDH, COX I, COX IV, PDHe1a, PDK2, PDK4, MCT1 and PFK) gels, and were subsequently transferred to a polyvinylidene difluoride membrane. Blotting was completed with commercially available antibodies for COX I, COX IV, LDH, mTOR, S6K1 (all Cell Signaling Technology, Danvers, MA), GAPDH, SIRT1 (Millipore, Billerica, MA), H3, PDK4, PFK (Abcam, Cambridge, MA), PDH (Invitrogen/Molecular Probes, Grand Island, NY), PDK2 (Abgent, San Diego, CA), and PGC-1 $\alpha$ (Calbiochem, San Diego, CA). The MCT1 antibody was a gift from from Dr. A. P. Halestrap (Dept. of Biochemistry, University of Bristol, Bristol, UK). Proteins were visualized by chemiluminescence detection, according to the manufacturer's instructions (Millipore, Billerica, MA). Blots were quantified using the Fluorochem HD2 System (Cell Biosciences, Santa Clara, CA). Samples were run in duplicate, except for nuclear extract samples, and equal protein loading was confirmed with GAPDH analysis and amido black staining.

An additional portion of muscle $(\sim 20 \mathrm{mg})$ was homogenized by hand for $30 \mathrm{~s}$ in glass hand homogenizers on ice and used to determine maximal citrate synthase (CS) activity. Total CS activity was measured spectrophotometrically at $37^{\circ} \mathrm{C}$ using the colormetric agent DTNB at $412 \mathrm{~nm}$.

\subsection{Statistical Analysis}

Changes in $\mathrm{VO}_{2}$ peak, aerobic power, Wingate mean power and Wingate peak power were analyzed using a one-way repeated measures ANOVA. Significant changes were subsequently analyzed using a Tukey's Post-Hoc test. All Western blot data were analyzed using paired
Student's $t$ tests. Significance was accepted at $p<0.05$ and data are presented as means \pm SD unless otherwise noted.

\section{RESULTS}

The heart rate and work rate response for a representative participant's first training session is presented in Figure 1(a). The group responses for perceived exertion, acute affect, and felt arousal are presented in Figures 1(b) and (c) respectively. Perceived exertion increased $(p<0.05)$ from baseline ( $7.8 \pm 0.6)$ following the 4 th interval (15.1 $\pm 0.5)$ and the 8th interval $(18.4 \pm 0.4)$ and remained elevated 10 minutes into recovery $(14.4 \pm 1.0)$. Acute affect decrease $(p<0.05)$ from baseline $(2.3 \pm 0.3)$ following both the 4th $(-0.4 \pm 0.7)$ and 8th interval $(-2.15$ $\pm 0.6)$ and remained lower than baseline during recovery $(0.1 \pm 0.9)$. Felt arousal was unchanged (baseline, $3.4 \pm$ 0.3 ; 4th interval, $3.6 \pm 0.5$; 8 th interval, $3.6 \pm 0.5$; recovery, $3.8 \pm 0.4)$.

Both relative (Figure 2(a)) and absolute (Table 1) $\mathrm{VO}_{2}$ peak were significantly elevated $(p<0.05)$ from baseline at both the mid- $(+9 \%)$ and post-training $(+19 \%)$ time points. These increases corresponded to significant increases $(p<0.05)$ in aerobic power (max power obtained during $\mathrm{VO}_{2}$ peak test; Table 1) from baseline (293 $\pm 24 \mathrm{~W})$ at both mid- $(327 \pm 21 \mathrm{~W},+12 \%)$ and posttraining $(334 \pm 31 \mathrm{~W},+14 \%)$. No change in Citrate Synthase maximal activity was observed following training $(p=0.17$; Figure 2(b)). Conversely, protein content of both COX I $(+27 \%)$ and COX IV $(+26 \%$; Figure 1(c)) were significantly elevated $(p<0.05)$ at the post-training time point. No change in total protein content of PDHela was observed $(p>0.05)$ while an increase in PDK2 $(+28 \% ; p=0.08)$, but not PDK4 $(+13 \% ; p=0.20)$ protein was observed (Figure 2(c)). GAPDH was used as a loading control with no significant change from baseline to post-training observed (see Figure 2(e) for representative blot).

Whole-muscle PGC-1 $\alpha$ was significantly $(p<0.05)$

Table 1. Participant characteristics before (pre), after 2 wks (mid), and after (post) 4 wks of training.

\begin{tabular}{cccc}
\hline & Pre- & Mid- & Post- \\
\hline Age, years & $20.6 \pm 1.6$ & & \\
Height $(\mathrm{cm})$ & $182 \pm 7$ & & \\
Weight $(\mathrm{kg})$ & $79.9 \pm 5.6$ & $79.2 \pm 5.2$ & $80.5 \pm 4.7$ \\
$\begin{array}{c}\mathrm{VO} \text { peak } \\
(\mathrm{mL} / \mathrm{min})\end{array}$ & $3182 \pm 227$ & $3455 \pm 192^{*}$ & $3786 \pm 438^{* \dagger}$ \\
$\begin{array}{c}\text { Peak aerobic } \\
\text { power (Watts) }\end{array}$ & $293 \pm 24$ & $327 \pm 21^{*}$ & $334 \pm 31^{*}$ \\
\hline
\end{tabular}

Values are mean $\pm \mathrm{SD}$; ${ }^{*}$ Significantly different $(p<0.05)$ than pre-training. 'Significantly different $(p<0.05)$ than mid-training. 

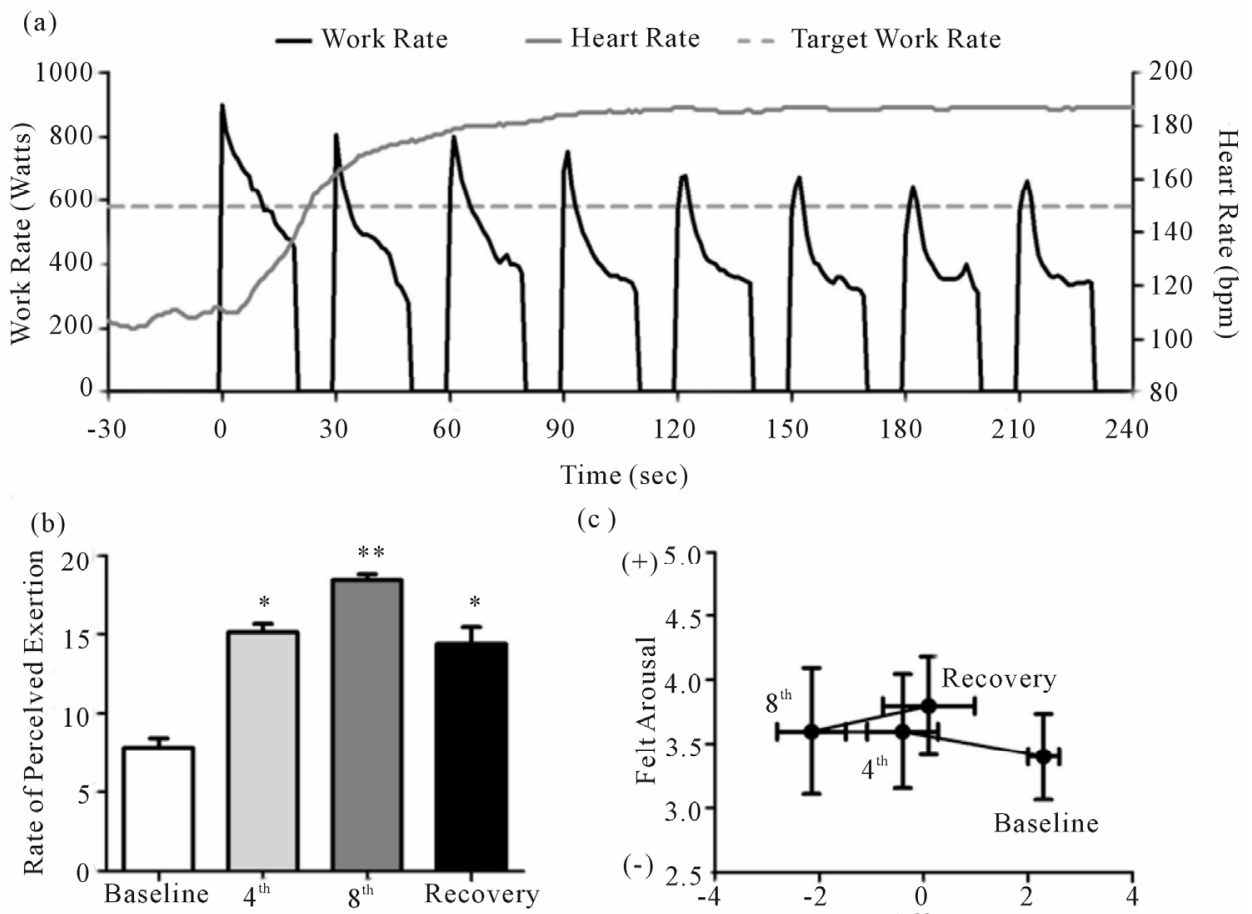

(c)

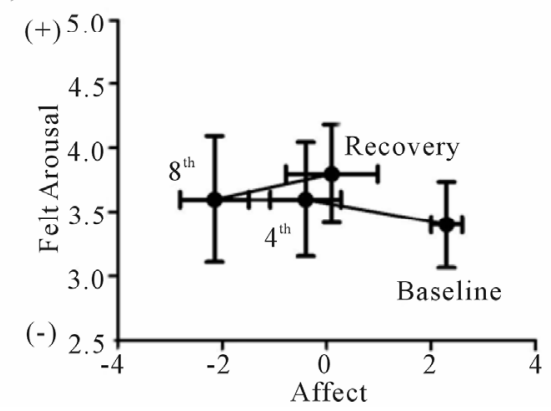

Figure 1. Heart rate and work rate response for a representative training session (a). Group responses for rate of perceived exertion (b), acute affect (c), and felt arousal (c). ${ }^{*}$ Significantly ( $p<$ $0.05)$ different from baseline. ${ }^{* *}$ Significantly $(p<0.05)$ different from baseline and 4 th interval.

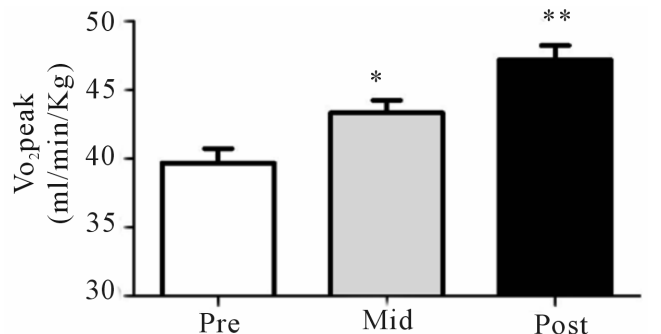

(a)

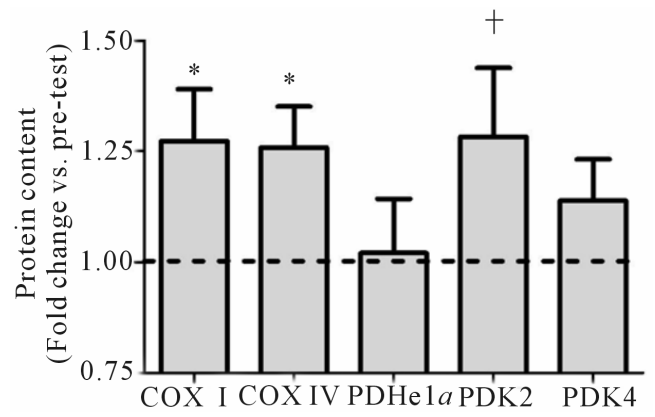

(c)

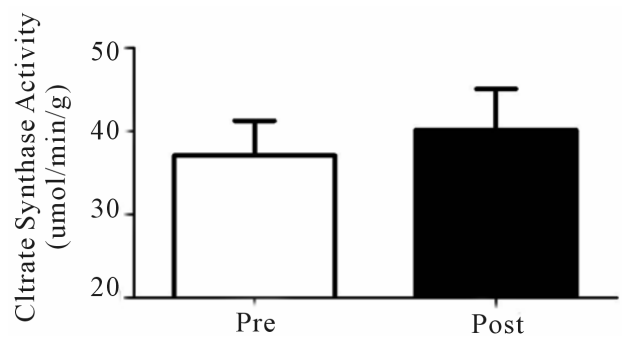

(b)

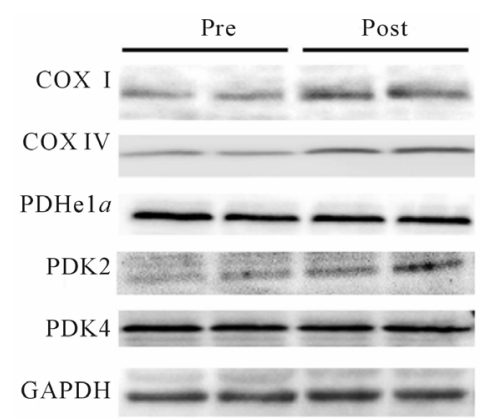

(d)

Figure 2. Relative $\mathrm{VO}_{2}$ peak (a) before training (Pre), following 2 wks of training (Mid) and following completion of 4 wks of training (Post), (b) Citrate Synthase maximal activity (umol $/ \mathrm{min} / \mathrm{g}$ ), (c) COX I, COX IV, PDHela, PDK2 and PDK4 before (Pre) and after (Post) 4 wks of Tabata training and representative blots for an individual participant are shown (d). ${ }^{*}$ Significantly $(p<0.05)$ different from Pre. ${ }^{\dagger}$ Significantly difference $(p=0.08)$ from pre. ${ }^{*}$ Significantly $(p<0.05)$ different from Pre and Mid. Cytochrome $c$ oxidase subunit I (COX I), cytochrome $c$ oxidase subunit IV (COX IV), glyceraldehyde 3-phosphate dehydrogenase (GAPDH), pyruvate dehydrogenase (PDH) kinase (PDK2 and 4). 
elevated $(+19 \%)$ following training. No significant changes were observed in the whole-muscle contents of SIRT1, mTOR, or S6K1 (Figure 3(a)). As with mitochondrial protein, equal loading was confirmed using GAPDH for all regulators of mitochondrial content (see Figure 3(b) for representative blot). Due to tissue limitations, we were only able to perform nuclear extraction on 3-paired samples. In these samples, PGC-1 $\alpha$ was elevated in the post-training samples when expressed as arbitrary units $(+46 \%, p<0.05)$ and when expressed relative to our nuclear loading control $\mathrm{H} 3(+110 \%, p=$

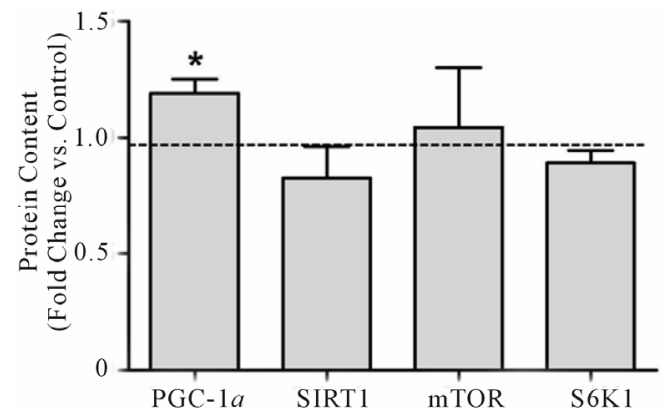

(a)
0.07).

Mean power during Wingate testing was also significantly elevated $(p<0.05)$ from baseline at both mid$(+9 \%)$ and post-training $(+11 \%$; Figure 4(a)), while peak power increased $(p<0.05)$ at the post-training time point only (pre, $975 \pm 114 \mathrm{~W}$; mid, $960 \pm 142 \mathrm{~W}$; post, $1095 \pm 166 \mathrm{~W},+12 \%$; Figure 4(b)). No change in either the glycolytic protein PFK ( $p>0.05$; Figure 4(c)) or the lactate transporter MCT1 $(p>0.05$; Figure 1(d)) was observed following training. Representative blots for PFK, MCT1 and GAPDH are presented in Figure 4(e).

Figure 3. Change in whole-muscle protein content from baseline to post training for PGC-1 $\alpha$, SIRT1, mTOR, and S6K1 (a). Representative blots for an individual participant are shown (b). "Significant increase $(p<0.05)$ from Pre. Mammalian target of rapamycin (mTOR), peroxisome proliferator-activated receptor $\gamma$ coactivator-1 alpha (PGC-1 $\alpha$ ), ribosomal protein S6 kinase (S6K1), silent mating-type information regulator 2 homolog 1 (SIRT1).

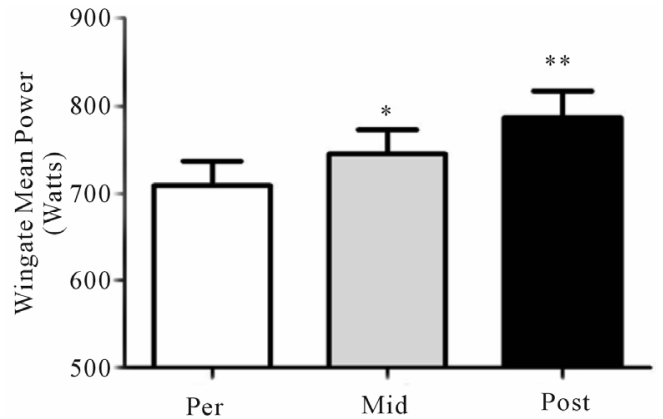

(a)

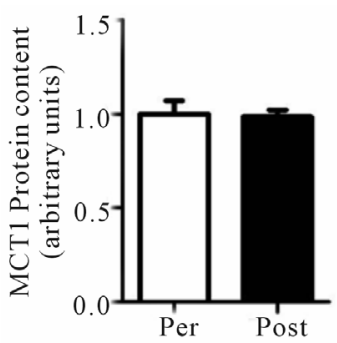

(c)

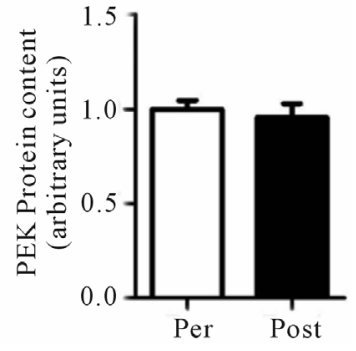

(d)

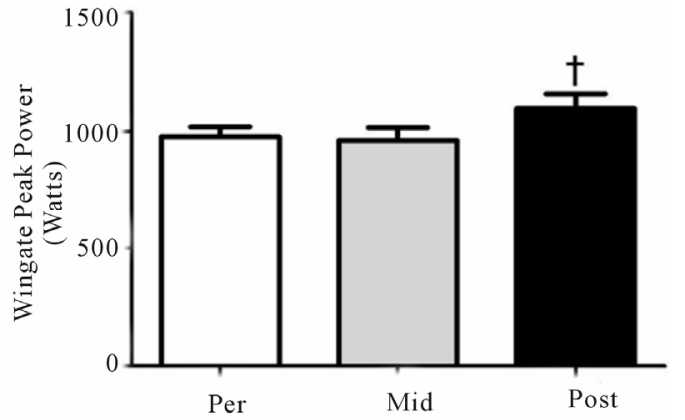

(b)

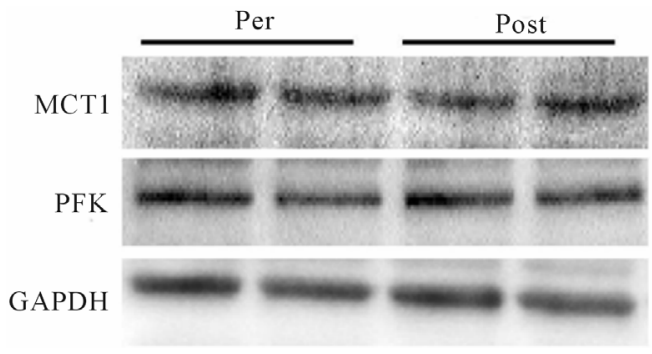

(e)

Figure 4. Wingate mean (a) and peak power (b) before training (Pre), following 2 wks of training (Mid) and following completion of 4 wks of training (Post). Fold change in MTC1 (c) and PFK (d) protein content following 4 wks of training, representative blots for an individual participant are shown (e). ${ }^{*}$ Significantly $(p<0.05)$ different from Pre. ${ }^{* *}$ Significantly $(p<0.05)$ different from Mid. ${ }^{\dagger}$ Significantly $(p<0.05)$ different from Pre. Phosphofructokinase (PFK), monocarboxylate transporter 1 (MTC1). 


\section{DISCUSSION}

This study evaluated changes in potential intramuscular mechanisms associated with increase in aerobic and anaerobic capacity induced by 4 wks of extremely lowvolume high-intensity cycling exercise. Consistent with a previous report [7] we observed increases in both aerobic and anaerobic capacity following 2 , and 4 wks of training utilizing the Tabata protocol. Increases in aerobic capacity coincided with increases in mitochondrial protein (COX I and IV), but not citrate synthase maximal activity. PGC- $1 \alpha$ protein was also elevated following training while no changes in SIRT1, mTOR or S6K1 were observed. The observed increase in anaerobic power was not accompanied by increases in either PFK or MCT1 protein content.

\subsection{Changes in Aerobic Capacity}

While other low volume HIT protocols have reported non-significant $[13,14]$ or small $(<10 \%)$ increases in aerobic capacity $[1,15]$ the current protocol induced relatively large increases in both $\mathrm{VO}_{2}$ peak $(+19 \%)$ and anaerobic performance $(+12 \%-14 \%)$. These findings confirm the results of Tabata et al. (1996) who reported elevated $\mathrm{VO}_{2}$ peak at both 3 and 6 wks of training, and demonstrate that increases in $\mathrm{VO}_{2}$ peak occur following 2 wks of training. Interestingly, while $\mathrm{VO}_{2}$ peak is traditionally believed to be determined by cardiac output, a recent report demonstrated increased $\mathrm{VO}_{2}$ peak without an accompanying increase in maximal cardiac output following treadmill sprint interval training [16]. These results, combined with our demonstration of elevated skeletal muscle mitochondrial protein, suggest that elevated aerobic capacity following low volume HIT may be result from peripheral adaptations.

While the current HIT protocol appears to be very time efficient, whether the absolute changes in mitochondrial content and aerobic capacity are greater than those observed following training protocols requiring a greater total training time commitment is of considerable interest. While we were unable to compare our results with another protocol in the current study, our results appear to compare favorably to previously published studies examining changes in aerobic capacity following training interventions of a similar duration (i.e. 4 weeks). Specifically, $\mathrm{VO}_{2}$ peak improved $9 \%$ and $\sim 6 \%$ following 4 weeks of high volume, steady state running [17] and cycling [18] respectively compared to $19 \%$ in the current study. While it is more difficult to compare changes in protein content and enzyme activity across studies, the increases in mitochondrial protein $(25 \%)$ appear in line with increases observed previously following endurance training and higher intensities of HIT $[1,2]$. Thus it would appear that the adaptations following Tabata training are at least comparable to endurance training. A future study performing a direct comparison of mitochondrial and aerobic adaptations following the low volume training protocol utilized in the current study, and higher volume endurance training would provide valuable insight into the efficacy of the Tabata protocol.

\subsection{Improved Mitochondrial Protein}

These results demonstrate that HIT cycle training requiring a total time commitment of 4 min per day $(<3$ min of exercise time), 4 days per week for 4 weeks can increase skeletal muscle mitochondrial protein content (COXI, COX IV). Surprisingly, these increases were not accompanied by an increase in maximal citrate synthase activity. While previous studies have demonstrated similar increases in mitochondrial protein with similarly low volume HIT $[1,2,5]$, the extremely high intensities associated with these protocols necessitates long recovery periods and thus training time commitment (20 - 30 min per session; 60 - 90 min per wk compared to only $16 \mathrm{~min}$ in the current study). We have also observed an increase $(p=0.08)$ in the protein content of PDK2, but not PDHe1 $\alpha$ or PDK4. These results are consistent with previous reports of no change in PDHel $\alpha$ following HIT [1] and the increase in PDK2, but not PDK4 typically observed following training [19]. Increases in PDK2 are associated with the inhibition of PDH and a greater reliance on FFA oxidation during exercise [19] suggesting that the Tabata protocol may also induce altered exercise substrate utilization, however, this adaptation has not been examined in humans.

Although the minimum volume of exercise required to induce muscle mitochondrial adaptations remains unclear, there appears to be a trade-off between volume and intensity whereby adaptations can be induced with a very low dose of exercise, provided the intensity is high. Our results indicate that high-intensity interval sessions lasting only $4 \mathrm{~min}$ are sufficient for promoting these adaptations in human skeletal muscle. Importantly, while the current results extend our knowledge of the minimum dose of HIT required, it is acknowledged that our findings fall in line with a growing body of literature that demonstrate a variety of intensities, durations, and work: rest ratios of HIT improve skeletal muscle mitochondrial protein content and fitness [20]. If HIT research is to continue to progress in a meaningful way there is a need for studies designed to optimize the beneficial adaptations of HIT while minimizing the safety concerns associated with supramaximal exercise [21]. The current results may serve as a starting point for this research, as, at present, the Tabata protocol appears to represent the most time efficient means of improving both skeletal muscle mitochondrial protein and aerobic and anaerobic performance. 


\subsection{Changes in Regulators of Mitochondrial Content}

PGC- $1 \alpha$ is a critical regulator of oxidative enzyme expression [22] and the activation of genes that encode for mitochondrial proteins [23] through its interaction with both nuclear and mitochondrial transcriptional factors [22,24,25]. Multiple investigators have identified increases in whole-muscle $[1,12,26]$ and nuclear $[3,12]$ content of PGC- $1 \alpha$ following HIT. Our results corroborate these findings, and provide the first evidence of the efficacy of the Tabata protocol at stimulating increases in whole-muscle PGC-1 $\alpha$ in human skeletal (Figure 2(a)). While tissue limitations restricted our ability to examine nuclear PGC- $1 \alpha$ content in the current study, the results from the 3 paired samples we were able to examine are consistent with previous studies observing exercise induced increases in nuclear PGC- $1 \alpha[3,12,27]$ and suggest that an increased abundance of PGC- $1 \alpha$ in the nucleus may contribute to the molecular adaptations induced by extremely low-volume HIT.

SIRT1, a deacetylase acting on PGC- $1 \alpha[28,29]$, has been suggested as a mediator of PGC- $1 \alpha$ activation [29, 30], expression [31], and nuclear sequestration [3]. The exact mechanisms by which SIRT1 contributes to exercise-mediated mitochondrial biogenesis are unclear [12, $26]$ and both increases $[3,31]$ and decreases $[12,26,32]$ in whole muscle SIRT1 protein content have been reported following exercise training. While no change in SIRT1 protein was observed in the current study, we have previously hypothesized that nuclear SIRT1 activity, rather than protein content per se, contributes to activation of PGC-1 $\alpha$ in human skeletal muscle [12]. Unfortunately, we were unable to measure nuclear SIRT1 activity in the current study due to tissue limitations. Despite this, our results are consistent with a growing body of literature that suggests an increase in whole muscle SIRT1 protein is not required for enhanced muscle mitochondrial protein following HIT [33].

Exercise-induced increases in mitochondrial and myofibrillar proteins are also hypothesized to be reliant on transient increases in signalling proteins in the mTOR/ S6K1 pathway [34,35]. Wilkinson et al. (2008) reported acute activation of mTOR and S6K1 following endurance and resistance exercise in trained and untrained individuals, however, little is known regarding regulation of the mTOR/S6K1 pathway in response to interval training. We observed no significant changes in wholemuscle mTOR or S6K1 content $72 \mathrm{hr}$ following the Tabata training protocol (Figure 2(a)). While our results suggest that neither mTOR nor S6K1 protein are upregulated by HIT, whether or not increased activity and/ or signalling responsiveness of the mTOR pathway contributes to increased translation of proteins involved in adaptations to HIT remains a possibility worthy of future research.

\subsection{Mechanisms of Increased Anaerobic Capacity}

Consistent with the original report by Tabata et al. (1996) we have observed improvements in anaerobic capacity following both 2 and 4 weeks of training. Increases in anaerobic performance are typically associated with improved content of glycolytic protein [9] and increased capacity for lactate transport out of exercise muscle [10]. Surprisingly, we have not observed increases in either PFK or MCT1 protein content. While others have also failed to observe an increase in MCT1 and MCT4 following HIT [36] it is possible that MCT4 increased following training in the current study, resulting in improved lactate export. While we were unable to measure MCT4 this explanation seems unlikely as MCT1 and MCT4 typically change in concert $[5,36]$. Alternatively, the observed increases in anaerobic power may be a result of improved muscle buffering capacity. While increases in buffering capacity have been observed following HIT training [2], no changes in buffering capacity have also been reported following HIT [36]. The mechanisms underlying the increases in anaerobic power induced by the Tabata protocol, and HIT in general, remain unclear.

\section{REFERENCES}

[1] Burgomaster, K.A., Howarth, K.R., Phillips, S.M., Rakobowchuk, M., MacDonald, M.J., McGee, S.L. and Gibala, M.J. (2008) Similar metabolic adaptations during exercise after low volume sprint interval and traditional endurance training in humans. The Journal of Physiology, 586, 151-160.

http://dx.doi.org/10.1113/jphysiol.2007.142109

[2] Gibala, M.J., Little, J.P., van Essen, M., Wilkin, G.P., Burgomaster, K.A., Safdar, A., Raha, S. and Tarnopolsky, M.A. (2006) Short-term sprint interval versus traditional endurance training: Similar initial adaptations in human skeletal muscle and exercise performance. The Journal of Physiology, 575, 901-911.

http://dx.doi.org/10.1113/jphysiol.2006.112094

[3] Little, J.P., Safdar, A., Wilkin, G.P., Tarnopolsky, M.A. and Gibala, M.J. (2010) A practical model of low-volume high-intensity interval training induces mitochondrial biogenesis in human skeletal muscle: Potential mechanisms. The Journal of Physiology, 588, 1011-1022. http://dx.doi.org/10.1113/jphysiol.2009.181743

[4] Terada, S., Yokozeki, T., Kawanaka, K., Ogawa, K., Higuchi, M., Ezaki, O. and Tabata, I. (2001) Effects of high-intensity swimming training on GLUT-4 and glucose transport activity in rat skeletal muscle. Journal of Applied Physiology, 20, 2019-2024.

[5] Burgomaster, K.A., Cermak, N.M., Phillips, S.M., Ben- 
ton, C.R., Bonen, A. and Gibala, M.J. (2007) Divergent response of metabolite transport proteins in human skeletal muscle after sprint interval training and detraining. AJP: Regulatory, Integrative and Comparative Physiology, 292, R1970-R1976.

[6] McRae, G., Payne, A., Zelt, J.G.E., Scribbans, T.D., Jung, M.E., Little, J.P. and Gurd, B.J. (2012) Extremely low volume, whole-body aerobic-resistance training improves aerobic fitness and muscular endurance in females. Applied Physiology, Nutrition, and Metabolism, 37, 11241131. http://dx.doi.org/10.1139/h2012-093

[7] Tabata, I., Nishimura, K., Kouzaki, M., Hirai, Y., Ogita, F., Miyachi, M. and Yamamoto, K. (1996) Effects of moderate-intensity endurance and high-intensity intermittent training on anaerobic capacity and $\mathrm{VO}_{2 \max }$. Medicine \& Science in Sports \& Exercise, 28, 1327-1330. http://dx.doi.org/10.1097/00005768-199610000-00018

[8] Terada, S., Tabata, I. and Higuchi, M. (2004) Effect of high-intensity intermittent swimming training on fatty acid oxidation enzyme activity in rat skeletal muscle. The Japanese Journal of Physiology, 54, 47-52. http://dx.doi.org/10.2170/jjphysiol.54.47

[9] Roberts, A.D., Billeter, R. and Howald, H. (1982) Anaerobic muscle enzyme changes after interval training. International Journal of Sports Medicine, 3, 18-21. http://dx.doi.org/10.1055/s-2008-1026055

[10] Green, H., Halestrap, A., Mockett, C., O'Toole, D., Grant, S. and Ouyang, J. (2002) Increases in muscle MCT are associated with reductions in muscle lactate after a single exercise session in humans. American Journal of Physiology, Endocrinology and Metabolism, 282, E154-E160.

[11] Bergstrom, J. (1975) Percutaneous needle biopsy of skeletal muscle in physiological and clinical research. Scandinavian Journal of Clinical \& Laboratory Investigation, 35, 609-616.

http://dx.doi.org/10.3109/00365517509095787

[12] Gurd, B.J., Yoshida, Y., McFarlan, J.T., Holloway, G.P., Moyes, C.D., Heigenhauser, G.J.F., Spriet, L. and Bonen, A. (2011) Nuclear SIRT1 activity, but not protein content, regulates mitochondrial biogenesis in rat and human skeletal muscle. AJP: Regulatory, Integrative and Comparative Physiology, 301, R67-R75.

[13] Burgomaster, K.A., Heigenhauser, G.J.F. and Gibala, M.J. (2006) Effect of short-term sprint interval training on human skeletal muscle carbohydrate metabolism during exercise and time-trial performance. Journal of Applied Physiology, 100, 2041-2047. http://dx.doi.org/10.1152/japplphysiol.01220.2005

[14] Burgomaster, K.A., Hughes, S.C., Heigenhauser, G.J.F., Bradwell, S.N. and Gibala, M.J. (2005) Six sessions of sprint interval training increases muscle oxidative potential and cycle endurance capacity in humans. Journal of Applied Physiology, 98, 1985-1990. http://dx.doi.org/10.1152/japplphysiol.01095.2004

[15] Hazell, T.J., MacPherson, R.E.K., Gravelle, B.M.R. and Lemon, P.W.R. (2010) 10 or 30 -s sprint interval training bouts enhance both aerobic and anaerobic performance. European Journal of Applied Physiology, 110, 153-160. http://dx.doi.org/10.1007/s00421-010-1474-y
[16] MacPherson, R.E.K., Hazell, T.J., Olver, T.D., Paterson, D.H. and Lemon, P.W.R. (2011) Run sprint interval training improves aerobic performance but not maximal cardiac output. Medicine \& Science in Sports \& Exercise, 43, 115-122. http://dx.doi.org/10.1249/MSS.0b013e3181e5eacd

[17] Daniels, J.T., Yarbrough, R.A. and Foster, C. (1978) Changes in $\mathrm{VO}_{2}$ max and running performance with training. European Journal of Applied Physiology and Occupational Physiology, 39, 249-254. http://dx.doi.org/10.1007/BF00421448

[18] Meredith, C.N., Frontera, W.R., Fisher, E.C., Hughes, V.A., Herland, J.C., Edwards, J. and Evans, W.J. (1989) Peripheral effects of endurance training in young and old subjects. Journal of Applied Physiology, 66, 2844-2849.

[19] LeBlanc, P.J., Peters, S.J., Tunstall, R.J., Cameron-Smith, D.D. and Heigenhauser, G.J.F. (2004) Effects of aerobic training on pyruvate dehydrogenase and pyruvate dehydrogenase kinase in human skeletal muscle. The Journal of Physiology, 557, 559-570. http://dx.doi.org/10.1113/jphysiol.2003.058263

[20] Gibala, M.J., Little, J.P., MacDonald, M.J. and Hawley, J.A. (2012) Physiological adaptations to low-volume, high-intensity interval training in health and disease. The Journal of Physiology, 590, 1077-1084. http://dx.doi.org/10.1113/jphysiol.2011.224725

[21] Gaesser, G.A. and Angadi, S.S. (2011) High-intensity interval training for health and fitness: Can less be more? Journal of Applied Physiology, 111, 1540-1541. http://dx.doi.org/10.1152/japplphysiol.01237.2011

[22] Puigserver, P. and Spiegelman, B.M. (2003) Peroxisome proliferator-activated receptor-gamma coactivator 1alpha (PGC-1alpha): Transcriptional coactivator and metabolic regulator. Endocrine Reviews, 24, 78-90. http://dx.doi.org/10.1210/er.2002-0012

[23] Scarpulla, R.C. (2002) Nuclear activators and coactivators in mammalian mitochondrial biogenesis. Biochimica et Biophysica Acta, 1576, 1-14. http://dx.doi.org/10.1016/S0167-4781(02)00343-3

[24] Knutti, D. and Kralli, A. (2001) PGC-1, a versitile coactivator. Trends in Endocrinology \& Metabolism, 12, 360365. http://dx.doi.org/10.1016/S1043-2760(01)00457-X

[25] Safdar, A., Little, J.P., Stokl, A.J., Hettinga, B.P., Akhtar, M. and Tarnopolsky, M.A. (2011) Exercise increases mitochondrial PGC-1 content and promotes nuclear-mitochondrial cross-talk to coordinate mitochondrial biogenesis. Journal of Biological Chemistry, 286, 10605-10617. http://dx.doi.org/10.1074/jbc.M110.211466

[26] Gurd, B.J., Perry, C.G.R., Heigenhauser, G.J.F., Spriet, L.L. and Bonen, A. (2010) High-intensity interval training increases SIRT1 activity in human skeletal muscle. Applied Physiology, Nutrition, and Metabolism, 35, 350357. http://dx.doi.org/10.1139/H10-030

[27] Wright, D.C., Han, D.-H., Garcia-Roves, P.M., Geiger, P.C., Jones, T.E. and Holloszy, J.O. (2007) Exercise-induced mitochondrial biogenesis begins before the increase in muscle PGC-1 expression. Journal of Biological Chemistry, 282, 194-199. http://dx.doi.org/10.1074/jbc.M606116200 
[28] Cantó, C., Gerhart-Hines, Z., Feige, J.N., Lagouge, M., Noriega, L., Milne, J.C., Elliott, P.J., Puigserver, P. and Auwerx, J. (2009) AMPK regulates energy expenditure by modulating NAD + metabolism and SIRT1 activity. Nature, 458, 1056-1060. http://dx.doi.org/10.1038/nature07813

[29] Gerhart-Hines, Z., Rodgers, J.T., Bare, O., Lerin, C., Kim, S.H., Mostoslavsky, R., Alt, F.W., Wu, Z. and Puigserver, P. (2007) Metabolic control of muscle mitochondrial function and fatty acid oxidation through SIRT1/PGC- $1 \alpha$. EMBO Journal, 26, 1913-1923. http://dx.doi.org/10.1038/sj.emboj.7601633

[30] Nemoto, S., Fergusson, M.M. and Finkel, T. (2005) SIRT1 functionally interacts with the metabolic regulator and transcriptional coactivator PGC-1. Journal of Biological Chemistry, 280, 16456-16460. http://dx.doi.org/10.1074/jbc.M501485200

[31] Suwa, M., Nakano, H., Radak, Z. and Kumagai, S. (2008) Endurance exercise increases the SIRT1 and peroxisome proliferator-activated receptor $\gamma$ coactivator- $1 \alpha$ protein expressions in rat skeletal muscle. Metabolism, 57, 986998. http://dx.doi.org/10.1016/j.metabol.2008.02.017

[32] Gurd, B.J., Yoshida, Y., Lally, J., Holloway, G.P. and Bonen, A. (2009) The deacetylase enzyme SIRT1 is not associated with oxidative capacity in rat heart and skeletal muscle and its overexpression reduces mitochondrial biogenesis. The Journal of Physiology, 587, 1817-1828. http://dx.doi.org/10.1113/jphysiol.2008.168096

[33] Gurd, B.J., Little, J.P. and Perry, C.G.R. (2012) Last word on viewpoint: Does SIRT1 determine exercise-induced skeletal muscle mitochondrial biogenesis: Differences between in vitro and in vivo experiments? Journal of Applied Physiology, 112, 931-931. http://dx.doi.org/10.1152/japplphysiol.00078.2012

[34] Jastrzebski, K., Hannan, K.M., Tchoubrieva, E.B., Hannan, R.D. and Pearson, R.B. (2007) Coordinate regulation of ribosome biogenesis and function by the ribosomal protein S6 kinase, a key mediator of $\mathrm{mTOR}$ function. Growth Factors, 25, 209-226. http://dx.doi.org/10.1080/08977190701779101

[35] Wilkinson, S.B., Phillips, S.M., Atherton, P.J., Patel, R., Yarasheski, K.E., Tarnopolsky, M.A. and Rennie, M.J. (2008) Differential effects of resistance and endurance exercise in the fed state on signalling molecule phosphorylation and protein synthesis in human muscle. The Journal of Physiology, 586, 3701-3717. http://dx.doi.org/10.1113/jphysiol.2008.153916

[36] Bishop, D., Edge, J., Thomas, C. and Mercier, J. (2008) Effects of high-intensity training on muscle lactate transporters and postexercise recovery of muscle lactate and hydrogen ions in women. AJP: Regulatory, Integrative and Comparative Physiology, 295, R1991-R1998. http://dx.doi.org/10.1152/ajpregu.00863.2007 